\title{
Diagnostic and therapeutic procedures in gout
}

\author{
Corresponding author: \\ Rafał Wojciechowski, Department \\ of Rheumatology and Connective \\ Tissue Diseases, \\ University Hospital No 2 \\ in Bydgoszcz, \\ e-mail: r.wojciechowski@wp.eu
}

Medical Research Journal 2019; Volume 4, Number 1, 95-98 10.5603/MRJ.a2019.0013 Copyright (C) 2019 Via Medica ISSN 2451-2591

\begin{abstract}
Gout, a rheumatic disease caused by crystals (crystal arthropathy), is a form of inflammatory arthritis caused by monosodium urate depositing in the synovial fluid and as time goes by outside the joints as well (in other tissues and organs).Gout attacks are sudden and often result from a dietary mistake. In 2015 the European and American Rheumatological Associations (EULAR and ACR) published joint classification criteria for gout. The criteria involve gout-specific clinical symptoms, irregular results of laboratory tests and lesions visible in imaging tests. The "golden standard" of diagnostics still remains the presence of uric acid crystals in a sample of synovial fluid, the contents of the bursa and of the gouty tophus. The course of treatment for patients with gout depends on the stage of the disease, but it comes down to implementing various forms of preventing hyperuricemia by modifying the patient's lifestyle and diet, reducing risk factors (such as overweight and obesity) and pharmacological treatment, both in-between and during attacks. Key words: gout, arthritis, crystal arthropathy, hyperuricemia, flares of joint inflammation, tophaceous gout, gouty tophi, Colchicine
\end{abstract}

Med Res J 2019; 4 (2): 95-98

\section{Introduction}

Gout, a rheumatic disease caused by crystals (crystal arthropathy), is a form of inflammatory arthritis caused by monosodium urate depositing in the synovial fluid and as time goes by outside the joints as well (in other tissues and organs).

Gout is believed to be the most common type of inflammatory arthropathy in men after 40 . It is 3 times more common in man than in women and the rate of incidence increases with age. It is also more common in developed countries, due to unhealthy lifestyle.

\section{The course of the disease}

Clinical symptoms of the disease are a result of hyperuricemia, which is an elevated level of uric acid. This happens due to a lack of balance between its supplied and excreted amount. Uric acid is the end product of the metabolic breakdown of purines, which may originate from food or internal metabolic processes. The overall amount of uric acid in the human body is estimated at approx. $1200 \mathrm{mg}$, which translates into a blood serum concentration of $5.2 \mathrm{mg} / \mathrm{dl}(310 \mu \mathrm{mol} / \mathrm{L})$ in males, $4.0 \mathrm{mg} / \mathrm{dl}(240 \mu \mathrm{mol} / \mathrm{L})$ in pre-menopause females, and
$4.7 \mathrm{mg} / \mathrm{dl}(280 \mu \mathrm{mol} / \mathrm{L})$ in post-menopause females. The daily renal excretion rate is $700-800 \mathrm{mg}$. [1]

The natural course of the disease can be characterized by four stages:

1. Asymptomatic hyperuricemia

2. Flares of joint inflammation (acute attacks of gout)

3. Interparoxysmal periods

4. Chronic gout (tophaceous gout) with symptoms of multi-joint inflammation and the formation of socalled gouty tophi.

The most recent modification of the course of gout classifies it into the following stages:

1. A great risk of gout - corresponds to the hyperuricemia stage, both without clinical symptoms and without typical irregularities in imaging tests or the presence of uric acid crystals in microscopic tests.

2. Asymptomatic hyperuricemia - a phase which is characterized by a lack of symptoms with the presence of monosodium urate in imaging and microscopic tests.

3. Gout attacks

4. Tophaceous gout - chronic arthritis with inflammation visible in imaging tests in the form of joint destruction and bone erosions.

The course of treatment for patients with gout depends on the stage of the disease, but it comes down 
to implementing various forms of preventing hyperuricemia by modifying the patient's lifestyle and diet, reducing risk factors (such as overweight and obesity) and pharmacological treatment, both in-between and during attacks. [2]

\section{Clinical symptoms}

Gout attacks are sudden and often result from a dietary mistake (too many purines and fructose, excessive portions, alcohol intake), substantive physical strain, fever, dehydration, effects of medication, an injury, or surgery.

The symptoms of an attack have all the characteristics of acute arthritis: intense pain, haphalgesia, articular hydrops, and anasarca. The skin around the joint is red, warm and tense. The first attack of gout usually affects the first metatarsophalangeal joint (podagra) and consecutive attacks may also affects this area. The inflammation may also affect other regions: ankle joints, knee joints, and rarely the joints in the upper extremities. The attacks usually affect a single joint, multi-joint inflammation is rarer.

Laboratory tests show an accelerated ESR, elevated CRP levels and neutrocytosis. The level of uric acid in the blood serum increases significantly, but sometimes it can be surprisingly normal. Proteinuria and microscopic haematuria can be observed in urinalysis. An examination of the synovial fluid confirms inflammation and a microscopic evaluation in polarized light shows monosodium urate crystals. An ultrasound of the joints shows a double contour specific for gout, which is a result of deposits on the surface of the cartilage.

Diagnosing an attack of gout based on a thorough history, a physical examination and basic laboratory test result is usually not difficult. Implementing the right treatment significantly shortens the time-span of attacks, which can last several to a dozen days if untreated.

During a differential diagnosis of a gout attack in the form of acute inflammation of a single or multiple peripheral joints the following should be considered:

— infectious, that is septic, arthritis

- reactive arthritis

- peripheral joints affected by AS

- psoriatic arthritis

- other crystal arthropathies, such as calcium pyrophosphate dihydrate deposition disease (CPPD)

- an exacerbation of osteoarthritis.

\section{Diagnosis}

In 2015 the European and American Rheumatological Associations (EULAR and ACR) published joint classification criteria for gout. [3] They apply to patients who had at least 1 episode of peripheral arthritis or bursitis (oedema, pain, or tenderness). The criteria involve gout-specific clinical symptoms, irregular results of laboratory tests and lesions visible in imaging tests [X-ray, ultrasound and dual-energy CT (dual energy computed tomography - DECT)].

The "golden standard" of diagnostics still remains the presence of uric acid crystals in a sample of synovial fluid, the contents of the bursa and of the gouty tophus. If crystals are found, there is no need to apply the remaining criteria. If however, for various reasons, the examination of the synovial fluid is not possible, 2015 ACR-EULAR gout classification criteria need to be followed.

\section{ACR-EULAR Gout Classification Criteria [3, 4]}

Preliminary condition: 1 episode of peripheral arthritis or bursitis (oedema, pain, or tenderness).

The presence of uric acid crystals in the synovial fluid is sufficient to diagnose gout (if this condition is met, the patient can be diagnosed with gout without applying the criteria below)

The maximum score is 23 points. A score of $\geq 8$ point allows to diagnose the patient with gout. The Full ACR-EULAR Gout Classification Criteria are presented in Table 1.

\section{Treatment of gout}

The essence of gout treatment is to lower the level of uric acid to $<6 \mathrm{mg} / \mathrm{dl}$ by modifying the patient's lifestyle, implementing a proper diet and prescribing adequate treatment. Hyperuricemia prophylaxis, the difference between treating gout attacks, treating the disease in interparoxysmal (intercritical) periods and treating chronic gout should be considered.

\section{Lifestyle and diet}

- reducing body mass in overweight and obese patients

- a diet low in purines

- increasing the frequency of physical activity

- fighting addictions - reducing alcohol consumption, especially beer consumption, and nicotine intake

- primary and secondary prophylaxis of cardiovascular diseases, such as ischemic heart disease and circulatory failure, hypertension, peripheral artery disease, stroke, chronic kidney disease, and diabetes. 
Table 1. The full ACR-EULAR Gout Classification Criteria table with values for each item's score from web page: http:// goutclassificationcalculator.auckland.ac.nz/. The following website contains an algorithm of diagnostic procedures in accordance with the criteria and a calculator

\section{Entry Criterion $\rightarrow$ \\ (Only apply criteria below to those meeting this entry criterion)}

Sufficient Criterion $\rightarrow$

(If met, can classify as gout without applying criteria below)

Criteria

(to be used if Sufficient Criterion not met)

Score $\geq 8$ required for classification as gout

Pattern of joint/bursa involvement during symptomatic episode(s) ever

Characteristics of symptomatic episode(s) ever:

1.Erythema overlying affected joint (patient-reported or physician-observed

2.Can't bear touch or pressure to affected joint

3.Great difficulty with walking or inability to used affected joint

Clinical evidence of tophus: draining or chalk-like subcutaneous nodule under transparent skin, often with overlying vascularity, located in typical locations: joints, ears, olecranon bursae, finger pads, tendons (e.g. Achilles)

Serum urate: Measured by uricase method. Ideally should be scored at a time when the patient was not taking uratelowering treatment and patient was beyond 4 weeks of the start of an episode (i.e., during intercritical period); if practicable, retest under those conditions. The highest value irrespective of timinig should be scored.

\section{Synovial fluid analysis of a symptomatic (ever) joint or bursa}

Should be assessed by a trained observer

Imaging evidence of urate depositions in symptomatic (ever) joint or bursa: Ultrasound evidence of doublecontour sign or dual energy computed tomography (DECT) demonstrating urate deposition

Imaging evidence of gout-related joint damage:

Conventional radiography of the hands and/or feet demonstrate at least one erosion

TOTAL SCORE $=$
At least one episode of swelling, pain, or tenderness in a Yes No

Presence of MSU crystals in a symptomatic joint or bursa Yes (i.e., in synovial fluid) or tophus No

Categories

Score

Joint(s) or bursa(e) other than ankle, midfoot or $1^{\text {st }}$ MTP (or their involvement only as part of polyarticular presentation)

Ankle OR midfoot (as part of monoarticular or oligoarticular episode without MTP1 involvement)

MTP1 (as part of monoarticular or oligoarticular episode)

No characteristics

\section{0}

One characteristic

Two characteristics

Three characteristics

One typical episode

Recurrent typical episodes

Absent

Present

$<4 \mathrm{mg} / \mathrm{dl}$

$-4$

$4-<6 \mathrm{mg} / \mathrm{dl}$

0

$6-<8 \mathrm{mg} / \mathrm{dl}$

$8-<10 \mathrm{mg} / \mathrm{dl}$

$\geq 10 \mathrm{mg} / \mathrm{dl}$

Not done

Monosodium Urate (MSU) negative $\quad-2$

Absent OR Not done $\quad 0$

Present (either modality) $\quad 4$

Absent OR Not done $\quad 0$

Present

\section{Treating a gout attack} clude:

Except for medication, the treatment should also in-

— reducing the strain on the extremity with the affected joint

- the extremity should be comfortable and the joint area should be exposed in order to reduce tenderness
- using cold compresses

- proper hydration (it will allow to lower the concentration of uric acid). Pharmacotherapy:

- Colchicine - an alkaloid extracted from the autumn crocus plant (Colchicum autumnale), which reduces inflammation, although its effects are not yet fully known. The medication is taken during the 
first day of the attack in an initial dose of $1.0 \mathrm{mg}$, $0.5 \mathrm{mg}$ after an hour and, if need be, $0.5 \mathrm{mg}$ after 12 hours. In the following it is recommended to take $0.5 \mathrm{mg}$ three times a day, until the symptoms resolve. Contraindication for the use of colchicine are: renal or hepatic failure, pregnancy, blood disorders, heart function disorders, serious gastric and intestinal disorders. The medication shouldn't be combined with macrolides, statins, or cyclosporin. The most common adverse effects include gastro-intestinal symptoms, like stomach ache, nausea, and diarrhea. The medication can cause liver and kidney dysfunction and may be myelotoxic.

- Nonsteroidal anti-inflammatory drugs (NSAIDs) with the exception of aspirin (which increases the concentration of uric acid in blood serum) are alternatives to colchicine, if they are used in the maximum recommended therapeutic dose.

- Glucocorticosteroids (GCs) — recommended for patients with colchicine and NSAID contraindications, administered intra-articularly if possible. If this is difficult or impossible due to multi-joint inflammation, the medication is administered orally, initially at $0.5 \mathrm{mg}$ of prednisone per $\mathrm{kg}$ of body mass, usually over the course of 5 days with a gradual reduction of the dosage.

- Canakinumab - a human, monoclonal IL-1 $\beta$ antibody (unavailable in Poland) intended for patients with frequent attacks ( $\geq 3$ per year), who have colchicine, NSAID and GC contraindications [5].

Treatment of chronic gout

In the case of recurrent attacks ( $>2 /$ year), the presence of gouty tophi, chronic arthritis, and concomitant diseases, such as chronic nephropathy, hypertension, ischemic heart disease and heart failure a treatment targeted at lowering uric acid concentration should be considered. The aim is to achieve a uric acid concentration of $<6 \mathrm{mg} / \mathrm{dl}$ and in the case of gouty tophi and/or chronic arthritis in the course of gout, $<5 \mathrm{mg} / \mathrm{dl}$. The treatment should be started no earlier than 2 weeks after the attack has subsided, due to the risk of another attack.

- Allopurinol (a xanthine oxidase inhibitor) is a first line treatment to reduce uric acid concentration in blood serum, used at an initial dose of $100 \mathrm{mg} /$ day, which is gradually increased, usually every 4 weeks, until the optimal dose of $300-600 \mathrm{mg} /$ day is reached, which should reduce the uric acid concentration to $6 \mathrm{mg} / \mathrm{dl}$.

- Febuxostat - as an alternative to allopurinol, in case it's ineffective or causes adverse effects. The initial dose is $80 \mathrm{mg} /$ day and can be increased up to $120 \mathrm{mg} /$ day [6].

In case of a gout attack, patients who regularly take antihyperuricemic medication, like allopurinol (Allupol, Milurit) or febuxostat (Adenuric), should not stop their treatment, since this would increase the risk of future attacks. Due to the mechanism of medications that decrease the level of uric acid, which may cause a gout attack, in the initial phase of the treatment it should be taken in combination with colchicine at a dose of $0.5 \mathrm{mg} /$ day or with NSAIDs.

An addition or alternative to antihyperuricemics are urisuric medications, like probenecid or benzbromarone, which are unavailable in Poland. Losartan and fenofibrate are known to have a weaker urisuric effect and should be considered, especially for patients with hypertension and dyslipidemia. [7, 8]

\section{References}

1. Dalbeth N, So A. Hyperuricaemia and gout: state of the art and future perspectives. Ann Rheum Dis. 2010; 69(10): 1738-1743, doi: 10.1136/ard.2010.136218, indexed in Pubmed: 20858623.

2. Zimmermann-Górskal. Postepowanie diagnostyczno-terapeutyczne w chorobach wywoływanych przez kryształy. Reumatologia. 2012; 50: 177-180.

3. Neogi T, Jansen TL, Dalbeth N, et al. 2015 Gout classification criteria: an American College of Rheumatology/European League Against Rheumatism collaborative initiative. Ann Rheum Dis. 2015; 74(10): 1789-1798, doi: 10.1136/annrheumdis-2015-208237, indexed in Pubmed: 26359487.

4. Neogi T et al., Arthritis \& Rheumatology. 2015; 67(10): 2557-2568.

5. Maślińska M. Gout and calcium pyrophosphate dihydrate deposition disease. Reumatologia/Rheumatology Supplements. ; 2016: 105-109, doi: 10.5114/reum.2016.60011.

6. Bridgeman MB, Chavez B. Febuxostat for the treatment of gout. Expert Opin Pharmacother. 2015; 16(3): 395-398, doi: 10.1517/14656566.20 15.985588, indexed in Pubmed: 25556668.

7. Zimmermann-Górska I, Tuchocka-Kaczmarek A, Goncerz G. Rozpoznanie i leczenie dny moczanowej. Podsumowanie zaleceń międzynaorodwej grupy reumatologów w ramach inicjatywy 3e. Medycyna Praktyczna. 2014; 5: 61-65.

8. Zimmermann-Górska I. Dna moczanowa. Interna Szczeklika Gajewski P. (red. Medycyna Praktyczna, Kraków. ; 2018 